\title{
Caseous Calcification in the Mitral Annulus: An Unusual Presentation in a Young Patient
}

\author{
${ }^{1}$ Krishna P Gourav, ${ }^{2}$ Sunderlal Negi, ${ }^{3}$ Imran Bhat, ${ }^{4}$ Indranil Biswas, ${ }^{5}$ Srinath Damodaran, ${ }^{6}$ Rupesh Kumar
}

\begin{abstract}
Calcification of the mitral valve annulus is a chronic degenerative process commonly seen in the older age group. In developing countries like India, it can occur in later stages of rheumatic heart disease. Caseous calcification (CC) of the mitral annulus is a less described atypical variant of mitral valve calcification commonly reported in the basal area of the posterior mitral leaflet in elderly patients with degenerative diseases. The peculiarity of our case is the early age of development, i.e., $\mathrm{CC}$ had developed in a 15-year-old male patient with rheumatic heart disease diagnosed by echocardiography and helped in further management.
\end{abstract}

Keywords: Caseous calcification, Echocardiography, Mitral annulus calcification, Rheumatic heart disease

How to cite this article: Gourav KP, Negi S, Bhat I, Biswas I,Damodaran S, Kumar R. Caseous Calcification in the Mitral Annulus: An Unusual Presentation in a Young Patient.J Perioper Echocardiogr 2018;6(2):40-43.

\section{Source of support: Nil}

Conflict of interest: None

\section{INTRODUCTION}

Calcification in the mitral valve is a familiar echocardiographic finding. It is commonly seen in degenerative disorders, which are usually manifested in the older age group. In developing countries like India, rheumatic heart disease (RHD) is one of the common cause of mitral valve calcification (MVC). ${ }^{1,2}$ Caseous calcification of mitral annulus (CCMA) is a less described atypical variant of MVC commonly reported in the basal area of the posterior mitral leaflet at the junction between the left atrium and the left ventricle in elderly patients

\footnotetext{
1,3-5Senior Resident, ${ }^{2,6}$ Assistant Professor

${ }^{1}$ Department of Anesthesia and Intensive Care, Postgraduate Institute of Medical Education and Research, Chandigarh, India

${ }^{2-5}$ Department of Anesthesia, Postgraduate Institute of Medical Education and Research, Chandigarh, India

${ }^{6}$ Department of Cardiothoracic and Vascular Surgery, Postgraduate Institute of Medical Education and Research, Chandigarh,India
}

Corresponding Author: Krishna P Gourav, Senior Resident, Department of Anesthesia and Intensive Care, Postgraduate Institute of Medical Education and Research, Chandigar, India, e-mail: kpgmbbs777@gmail.com with degenerative diseases. ${ }^{3}$ We hereby report a case of CCMA in a 15-year-old boy with chronic RHD.

\section{CASE DESCRIPTION}

A 15-year-old male patient, a known case of RHD with severe mitral stenosis (MS) and severe aortic regurgitation (AR), presented to our hospital with New York Heart Association (NYHA) functional class III breathlessness, despite optimal medical management. The patient was planned for mitral and aortic valve replacement. The patient had no history of other comorbid conditions. The significant findings on physical examination were an irregularly irregular pulse with a rate of 105/ minute, apical mid-diastolic murmur, and bilateral basal crepitations. The routine investigations like hemogram, coagulation parameters, liver, and renal functions tests were within the normal range. A pre-induction two dimensional transthoracic echocardiographic (TTE) exam (M5S probe; GE vivid E9) in the operating room revealed thickened mitral, aortic and tricuspid valves with dilated left and right atrium. The color Doppler exam showed severe MS, severe mitral regurgitation (MR), severe AR and moderate tricuspid regurgitation (Fig. 1 and Video 1). In addition, a bright, round echo-dense structure with dimensions of $23 \mathrm{~mm} \times 15 \mathrm{~mm}$ was seen near the posterior

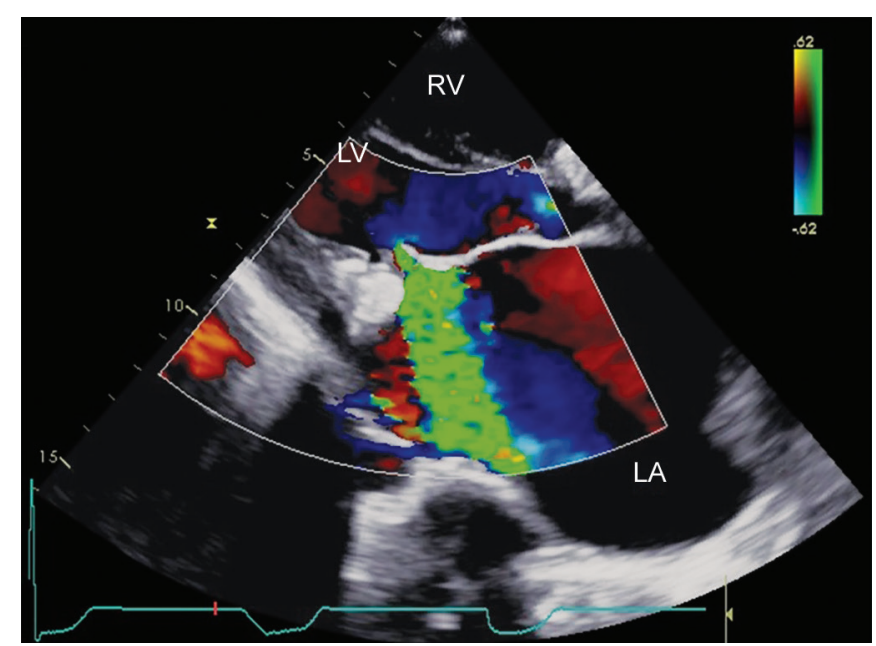

Fig. 1: Parasternal long axis view in transthoracic echocardiography showing a severe mitral regurgitation and an echo-dense mass located at the posterior mitral annulus. LA, left atrium; LV, left ventricle; RV, right ventricle 


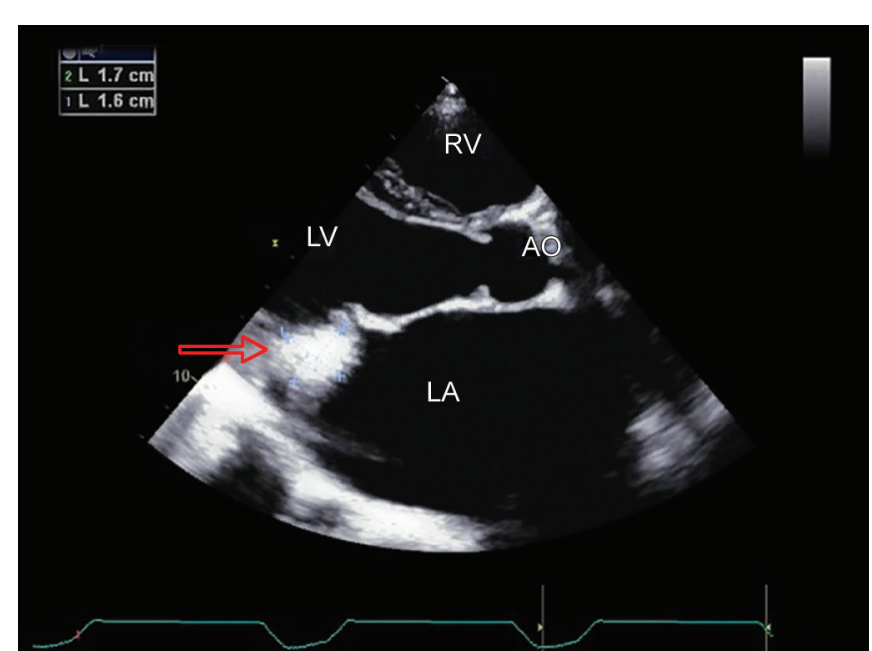

Fig. 2: Caseous calcification of posterior mitral annulus (parasternal aortic valve long axis view): Large echo-dense mass with smooth borders near posterior mitral annulus at the junction between left atrium (LA) and left ventricle (LV). RV, right ventricle

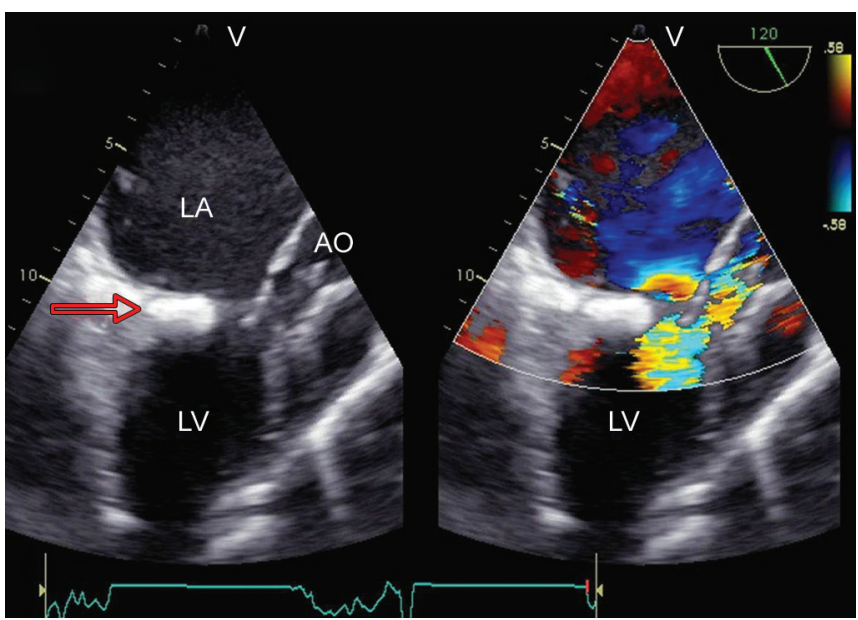

Fig. 4: Mid esophageal aortic valve long axis view in transesophageal echocardiography showing (A) a large round echo-dense mass near posterior mitral valve, (B) mitral stenosis and aortic regurgitation. AO, aorta; LA, left atrium; LV left ventricle

mitral annulus and extending into the left ventricle which was not described in the TTE exam done 4 months before the surgery (Figs 2 and 3 and Videos 2 and 3). The localized echo dense structure had distinct borders. Aortic and mitral valve replacement along with the excision of the echo dense structure was planned. Anesthesia was induced with intravenous fentanyl $100 \mathrm{mcg}$, intravenous propofol $(50 \mathrm{mg})$, intravenous ketamine $(50 \mathrm{mg})$, and intravenous vecuronium $(5 \mathrm{mg})$. After adequate muscle relaxation, the trachea was intubated with a $7.5 \mathrm{~mm}$ internal diameter endotracheal tube. The transesophageal echocardiography (TEE) (6VT probe; GE vivid E9), done after induction of anesthesia confirmed the findings of TTE (Fig. 4 and Video 4). Cardiopulmonary bypass (CPB) was initiated after standard aorto-bicaval cannulation. After achieving cardiac arrest, the mitral, tricuspid and aortic valves were inspected by the surgical team. All

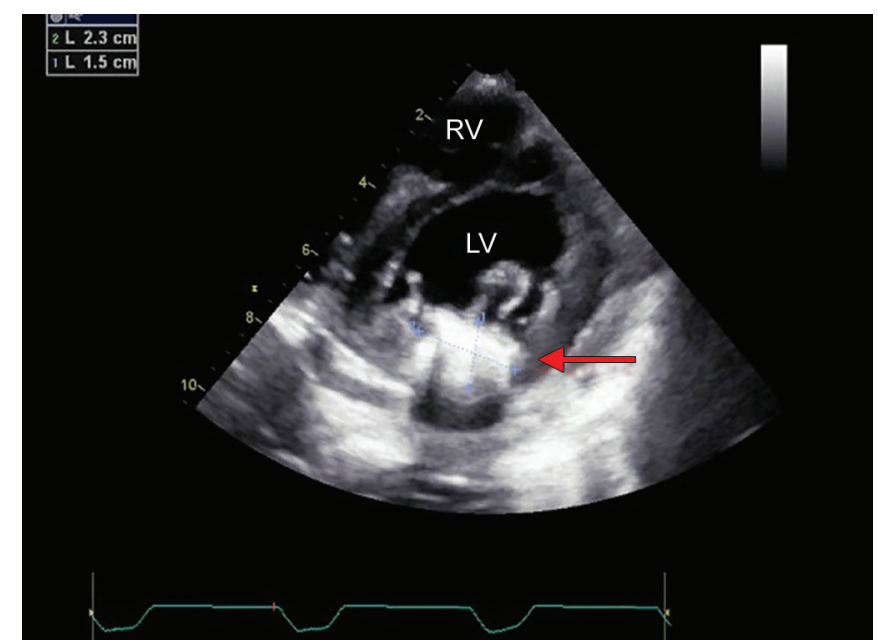

Fig. 3: Parasternal left ventricular basal short axis view showing a round, echo dense mass with smooth surface near posterior annulus of mitral valve. LV, left ventricle. $\mathrm{RV}$, right ventricle

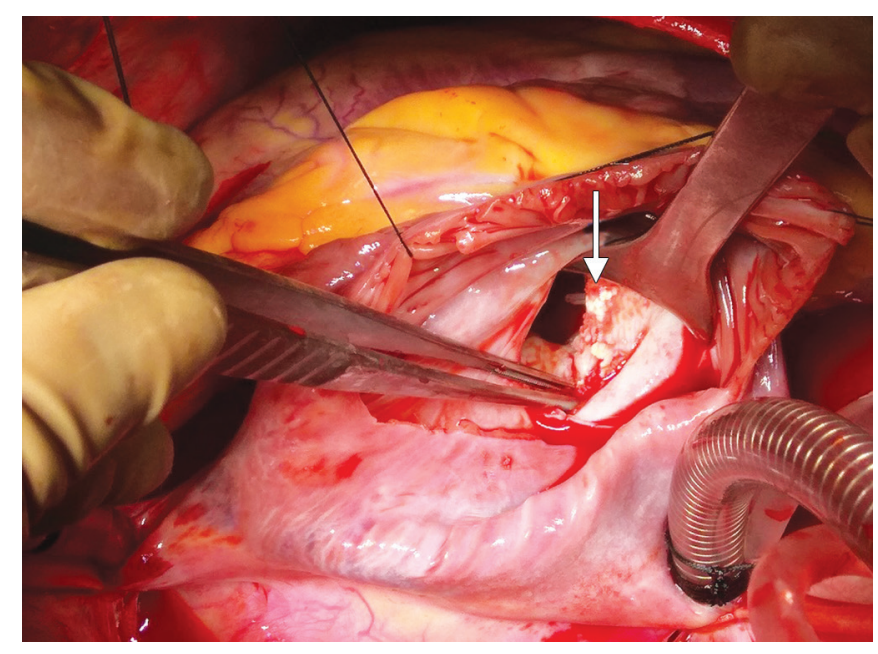

Fig. 5: Intraoperative image showing a thick, white cheesy material exuded from the mass during its excision

the three valves inspected were thickened. In addition, they found large, white mass measuring $25 \mathrm{~mm}$ X $17 \mathrm{~mm}$ near the posterior annulus of the mitral valve distorting the posterior mitral valve leaflet. A thick, white cheesy material exuded from the mass during its excision (Fig. 5). After replacing the aortic and mitral valves, the patient was successfully weaned off CPB using milrinone 0.3 $\mathrm{mcg} / \mathrm{kg} / \mathrm{minute}$ and noradrenaline $0.06 \mathrm{mcg} / \mathrm{kg} /$ minute The patient was transferred to the intensive care unit with stable hemodynamics. The patient's trachea was extubated after 5 hours of mechanical ventilation and was discharged from the hospital after 5 days of uneventful hospital stay. Histopathological examination of the mass revealed an amorphous eosinophilic material surrounded by lymphocytes and macrophages suggesting liquefactive necrosis. The culture of caseous material for bacteria and fungi were negative. 


\section{DISCUSSION}

Rheumatic heart disease is one of the leading acquired cardiac diseases in developing countries like India. ${ }^{4}$ The mitral valve is commonly involved in RHD followed by aortic valve and it is characterized by repeated inflammation with the fibrinous repair. The cardinal anatomical changes of valve include leaflet thickening, commissural fusion, shortening and thickening of chordae tendineae. ${ }^{5}$ Mitral annular calcification (MAC) is a chronic degenerative process and develops in $10 \%$ of general population. ${ }^{6,7}$ It can occur in various conditions like old age, chronic renal failure, rheumatic heart disease, hypertension, congenital metabolic syndromes and calcium-phosphorus metabolism abnormalities with a female preponderance. ${ }^{8} \mathrm{MAC}$ in RHD usually involves commissures and leaflet tissue, whereas, an extension to the annulus is a late occurrence. Rarely, MAC can develop caseous degeneration (also called as liquefaction necrosis) of internal material. This degenerative process can lead to the formation of CCMA in chronic cases of RHD. The degenerated material in CCMA is composed of a mixture of calcium, fatty acids, and cholesterol covered by a calcified envelope., 3 Pomerance 1 reported a $2.7 \%$ postmortem incidence of CCMA in patients older than 50 years with established MAC. Later on, two echocardiographic studies by Kronzen et al. ${ }^{10}$ and Harpez et al. ${ }^{3}$ reported a prevalence of $0.055 \%$ and $0.067 \%$ of CCMA in general population, respectively. Harpez et al. ${ }^{3}$ after subgroup analysis reported a $0.6 \%$ prevalence of CCMA in patients with mitral annular calcification. The actual incidence of CCMA in general population cannot be measured as the majority of the patients are asymptomatic and do not undergo echocardiographic evaluation. Till now it has been documented in various case reports and series in elderly patients. However, no such case has been reported in younger age group. In this index case of a 15-year-old male patient with chronic RHD, CCMA was suspected on echocardiography and confirmed on microscopic examination. To our knowledge, to date, no such case has been reported at such a young age.

The CCMA can be misinterpreted as tumor, abscess, vegetation, organized thrombus or calcification and can result in unnecessary surgery. ${ }^{11-13}$ On TTE and/or TEE it looks like a large, bright, round, echo-dense mass with well defined calcified smooth borders without acoustic shadowing artifacts and sometimes containing a central area of echo-lucent area suggesting caseous necrosis and is usually located near the posterior mitral valve annulus. Harpez et al. $^{3}$ reported 19 patients with CCMA. In 18 of those, the mass was located over the posterior region of mitral annulus at the junction between the left atrium and left ventricle, and in one patient it was located over the tricuspid annulus. The CCMA can be differentiated from mitral annular abscess by the presence of calcification in it whereas abscess lacks calcification and secondly, its usual location is near the posterior mitral annulus, however, an annular abscess is commonly located near the mitral aortic fibrosa. In the case of tumors, the interior echogenicity will be absent. ${ }^{14}$ In the index case, TTE revealed CCMA over the annulus of the posterior mitral leaflet and appeared as a round, bright, echo-dense mass with smooth calcified borders containing the central echo-lucent area.

\section{CONCLUSION}

Caseous calcification of the mitral valve is a rare variant of mitral annular calcification observed predominantly in the older age group. The peculiarity of our case is the early age at which the CCMA caseous necrosis of mitral annular calcification developed, has been echocardiographically diagnosed and documented in a 15 -year-old male patient with rheumatic heart disease. Thus a re-assessment and revision of preoperative diagnosis with meticulous perioperative echocardiography can help in formulating a surgical plan and/or modification of surgical steps.

\section{REFERENCES}

1. Pomerance A. Pathological and clinical study of calcification of the mitral valve ring. J Clin Pathol 1970;23:354-361.

2. Schott CR, Kotler MN, Parry WR, Segal BL. Mitral annular calcification. Clinical and echocardiographic correlations. Arch Intern Med 1977; 137: 1143-1150.

3. Harpaz D, Auerbach I, Vered Z, Motro M, Tobar A, RosenblattS. Caseous calcification of the mitral annulus: a neglected, unrecognized diagnosis. J Am Soc Echocardiogr. 2001;14:825-831.

4. Rajamannan NM, Nealis TB, Subramaniam M, et al. Calcified rheumatic valve neoangiogenesis is associated with vascular endothelial growth factor expression and osteoblast-like bone formation. Circulation. 2005 Jun 21;111(24):3296-3301.

5. Krapf L, Dreyfus J, Cueff C, Lepage L, Brochet E, Vahanian A et al. Anatomical features of rheumatic and non-rheumatic mitral stenosis: potential additional value of threedimensional echocardiography. Arch Cardiovasc Dis. 2013 Feb;106(2):111-115.

6. Fertman MH, Wolff L. Calcification of the mitral valve. Am Heart J 1946;31:580-589.

7. Allison MA, Cheung P, Criqui $\mathrm{MH}$, et al. Mitral and aortic annular calcification are highly associated with systemic calcified atherosclerosis. Circulation 1. 2006;113:861-866.

8. Abramowitz Y, Jilaihawi H, Chakravarty T, Mack MJ, Makkar RR. Mitral Annulus Calcification. J Am Coll Cardiol. 2015 Oct 27;66(17):1934-1941. 
9. Deluca G, Correale M, Leva R, Del Salvatore B, Gramenzi $\mathrm{S}$, Di Biase M. The incidence and clinical course of caseous calcification of the mitral annulus. J Am Soc Echocardiogr. 2008;21:828-833.

10 Kronzon I, Winer HE, Cohen ML. Sterile, caseous mitral annular abscess. J Am Coll Cardiol 1983;2:186-190.

11. Kautzner J, Vondráacek V, Jir ásek A, et al: Tumor-like mitral annular calcification with central liquefaction. Echocardiography 1993;10:459-463.

12. Borowski A, Korb H, Voth E, et al: Asymptomatic myocardial abscess. Thorac Cardiovasc Surg 1988;36:338-340.
13. Gilbert HM, Grodman R, Chung MH, et al: Sterile caseous mitral valve "abscess" mimicking infective endocarditis. Clin Infect Dis 1997;24:1015-1016.

14. García-Ibarrondo N, Lang RM. [Caseous calcification of the mitral annulus, a rare echocardiographic finding]. Rev Esp Cardiol. 2011 Sep;64(9):828-831.

15. Gramenzi S, Mazzola AA, Tagliaferro B, Protasoni G, Brusoni D, D'Aloya G, et al. Caseous calcification of the mitral annulus: unusual case of spontaneous resolution. Echocardiography. 2005;22:510-3. 